\title{
DEPRESSÃO MATERNA E IMPLICAÇÕES SOBRE O DESENVOLVIMENTO INFANTIL DO AUTISTA
}

\section{MATERNAL DEPRESSION AND IMPLICATIONS ON THE INFANT DEVELOPMENT OF AUTIST}

\author{
Cláudia Sanini ${ }^{1}$ \\ Evanisa Helena Maio de Brum ${ }^{2}$ \\ Cleonice Alves Bosa ${ }^{3}$
}

Sanini C; Brum EHM de; Bosa CA. Depressão materna e implicações sobre o desenvolvimento infantil do autismo. Rev. Bras. Cresc. e Desenv. Hum. 2010; 20(3) 809-815.

\section{Resumo}

Introdução: há evidências de intenso estresse nos cuidadores de crianças com autismo, especialmente nas mães, podendo ter, como uma de suas consequências, o desenvolvimento de um quadro depressivo materno. A depressão materna, por sua vez, pode afetar negativamente, tanto a mãe quanto a criança. Objetivo: descrever os aspectos implicados na depressão materna no contexto do autismo, bem como o impacto deste diagnóstico no desenvolvimento da criança com autismo. Método: foram consultadas as bases de dados do PsycINFO e SciELO. Utilizou-se como estratégias de busca no PsycINFO os descritores: autism OR infantile autism AND mother depression e no SciELO, Autism AND mother depression em todos os campos. Resultados: os artigos foram classificados quanto ao tipo: revisão, estudo de caso e estudo experimental $(\mathrm{N}=14)$. Foram considerados estudos de natureza qualitativa e quantitativa, escritos em português, inglês e espanhol. Os artigos listados de 1 a 3 foram utilizados para embasar a concepção de autismo e depressão materna. Conclusão: o diagnóstico de autismo pode afetar negativamente a saúde mental materna, o que por sua vez, pode impactar negativamente a interação com o filho, trazendo consequências para o desenvolvimento infantil. Intervenções destinadas a melhorar a saúde mental das mães e o desenvolvimento de crianças com autismo têm apresentado resultados positivos.

Palavras-chave: depressão materna; autismo e desenvolvimento infantil.

1 Mestre e Doutoranda do Programa de Pós-Graduação do Instituto de Psicologia da Universidade Federal do Rio Grande do Sul - Bolsista CAPES. E-mail: csanini71@terra.com.br

2 Psicóloga e Psicoterapeuta. Mestre em Saúde Mental/ULBRA, Doutora pelo Programa de Pós-Graduação do Instituto de Psicologia da Universidade Federal do Rio Grande do Sul - UFRGS e Coordenadora do Curso de Psicologia do Complexo de Ensino Superior de Cachoeirinha - CESUCA. Endereço: Rua Ramiro Barcelos, 2600 - Bairro Santa Cecília - Porto Alegre - RS - Brasil - CEP 90035-003. E-mail: evanisa.brum@gmail.com

3 Profa. Adjunta do Instituto de Psicologia da Universidade Federal do Rio Grande do Sul. Coordenadora do Núcleo de Estudos e Pesquisas em Transtornos do Desenvolvimento. Endereço: Rua Ramiro Barcelos, 2600 - Bairro Santa Cecília - Porto Alegre - RS - Brasil - CEP 90035-003. E-mail: cleobosa@uol.com.br

Correspondência para: Evanisa Helena Maio de Brum. Endereço: Rua Ramiro Barcelos, 2600 - Bairro Santa Cecília - Porto Alegre - RS - Brasil - CEP 90035-003. Telefone: (51) 33961040 e (51) 93589251. E-mail: evanisa.brum@gmail.com Trabalho realizado no Programa de Pós-Graduação do Instituto de Psicologia da Universidade Federal do Rio Grande do Sul UFRGS. 


\begin{abstract}
Introduction: there is evidence of intensive stress in caregivers of children with autism, especially mothers. One of its consequences may be the development of a depressive disorder. Maternal depression can adversely affect both the mother and the child. Objective: to describe the aspects involved in maternal depression in the context of autism and the impact of this diagnosis in child development. Method: it was consulted the databases PsycINFO and SciELO. As search strategies in PsycINFO the descriptors "autism OR infantile autism and mother depression" were used; in SciELO the terms were "autism and mother depression", in all fields. Results: The articles were classified according to the type: review, case study or experimental study $(\mathrm{N}=14)$. It was considered both qualitative and quantitative studies, in Portuguese, English and Spanish. The articles listed from 1 to 3 were used to base the conception of autism and maternal depression. Conclusion: the diagnosis of autism may adversely affect the maternal mental health of these children, and may negatively impact the mother-child interaction and child development. Interventions to improve the mental health of parents and development of children with autism have shown positive results.
\end{abstract}

Key words: maternal depression; autism and child development.

\section{INTRODUÇÃO}

A depressão materna caracteriza-se por sintomas como choro, confusão, ansiedade, humor lábil e deprimido, sendo que se inicia nos primeiros meses após o parto, podendo persistir por mais de um ano ${ }^{1}$.

Este tema tem sido alvo de inúmeros estudos, tendo em vista o aumento da prevalência deste quadro clínico, ${ }^{1-2}$ a dificuldade de caracterizá-lo, ${ }^{1}$ os danos causados à mãe, bem como o seu impacto no desenvolvimento infantil ${ }^{1,2}$.

Essa associação de fatores coloca em relevo a magnitude de um problema com consequências para a saúde pública e que necessita maior atenção dos clínicos, dos pesquisadores da área e dos formadores de políticas públicas, especialmente quando envolve alguma necessidade especial do filho, como no caso do autismo.

$\mathrm{O}$ autismo caracteriza-se por uma síndrome comportamental, que tem como sin- tomas básicos uma dificuldade nas interações sociais, um atraso na aquisição da fala e no uso não-comunicativo da mesma, e presença de um repertório restrito de atividades e interesses, com insistência obsessiva na rotina, limitando, assim, as atividades espontâneas e imaginativas ${ }^{3}$. Por definição (DSM-IV, 2002) os sintomas devem estar presentes antes dos três anos de idade, mas há evidências de preocupação parental com o desenvolvimento atípico da criança, ainda no primeiro ano de vida do bebê. Pois, nesta fase, a criança já começa a apresentar limitações que vão se evidenciando ao longo do seu desenvolvimento.

Há intenso estresse nos cuidadores de crianças com autismo, o que leva a consequências adversas tanto para o desenvolvimento infantil quanto para a família e, em especial, para a mãe.

Dessa forma, o objetivo é descrever os aspectos implicados na depressão materna no 
contexto do autismo, bem como o impacto desse diagnóstico no desenvolvimento infantil.

\section{MÉTODO}

Foram consultadas as bases de dados PsycINFO e SciELO no período de 1999 a 2010. No PsycINFO os termos utilizados para estratégia de busca foram autism OR infantile autism AND mother depression para encontrar os trabalhos que contivessem o termo em qualquer campo. No SciELO foram Autism AND mother depression para encontrar os trabalhos que contivessem o termo em qualquer campo.

A seleção dos textos foi realizada manualmente, sendo primeiro excluídos os livros, capítulos de livros, dissertações e teses e após considerados os artigos escritos em português, inglês e espanhol. Num terceiro momento os artigos foram classificados quanto ao tipo: revisão, estudo de caso e estudo experimental $(\mathrm{N}=14)$. Foram considerados estudos de natureza qualitativa e quantitativa. Os artigos de 1 a 3 foram utilizados para embasar a concepção de autismo e depressão materna.

\section{RESULTADOS}

Inicialmente foram encontrados, no PsycINFO, 28.946 textos, com a adição do termo mother depression reduziu-se o número para 64. Após, foram excluídos os livros, capítulos de livros, teses e dissertações, bem como os artigos que estivessem escritos em outras línguas que não português, inglês e espanhol. Dos 11 que permaneceram, nove eram estudos experimentais, um de revisão e um estudo de caso.

Na base de dados da SciELO foram encontrados 100 artigos. Com a adição do termo mother depression, nenhum artigo foi encontrado. Suprimindo-se o termo depression, reduziu-se para 8. Destes, foram excluídos os que não abordavam o tema da depressão materna, permanecendo apenas três, todos de revisão. Tanto na base de dados do PsyINFO quanto na SciELO nenhum artigo foi encontrado no ano de 2010, na época em que se procedeu a esta busca.

\section{DISCUSSÃO}

Há evidências na literatura de que, dentre os membros da família de uma criança com desenvolvimento atípico, as mães podem ser bastante atingidas emocionalmente, ${ }^{4}$ pois se deparam com a perda do filho imaginado por elas. Por isso, correm o risco de apresentar sentimentos como tristeza, frustração, ambivalência e negação, ${ }^{4}$ os quais podem alterar o relacionamento mãe-criança ${ }^{5,6}$. Além disso, ser mãe de uma criança com desenvolvimento atípico pode ser estressante, ${ }^{4}$ na medida em que existe uma sobrecarga adicional em todos os níveis: social, psicológico, financeiro e, também, nos cuidados com a criança ${ }^{5,6}$. Esta sobrecarga poderá variar de acordo com as características de cada criança e com a severidade da deficiência que ela apresenta, já que diferentes casos ori-

Tabela 1: Distribuição dos manuscritos nas bases de dados PsyINFO e SciELO no período de 1999 a 2010

\begin{tabular}{lccc}
\hline Tipo de manuscrito & SciELO & $\begin{array}{c}\text { Base de Dados } \\
\text { PsycINFO }\end{array}$ & Total \\
\hline Estudo experimental & - & 9 & 9 \\
Revisão & 3 & 1 & 4 \\
Caso clínico & - & 1 & 1 \\
\hline Total & 3 & 11 & 14 \\
\hline
\end{tabular}


Tabela 2: Síntese dos manuscritos que versaram sobre depressão materna no contexto do autismo no período de 1999 a 2010

\begin{tabular}{|c|c|}
\hline$\overline{\text { Hastings et al. }{ }^{4}}$ & $\begin{array}{l}\text { Pais e mães de crianças com autismo apresentam estresse e depressão, porém, } \\
\text { as mães relataram mais depressão quando comparadas aos pais. }\end{array}$ \\
\hline Fávero e Santos ${ }^{5}$ & $\begin{array}{l}\text { Nas famílias de crianças com autismo um dos principais tópicos } \\
\text { investigados é o estresse parental. }\end{array}$ \\
\hline Fernandes ${ }^{6}$ & $\begin{array}{l}\text { Cinco grandes temas são abordados em relação a famílias de crianças com } \\
\text { autismo, entre eles o estresse, as dificuldades emocionais e suporte. }\end{array}$ \\
\hline Bosa $^{7}$ & $\begin{array}{l}\text { A intervenção mais adequada no contexto do autismo depende da fase de } \\
\text { desenvolvimento da criança e o contexto familiar. }\end{array}$ \\
\hline Boyd $^{8}$ & $\begin{array}{l}\text { Baixos níveis de suporte social foram os preditores mais fortes para depressão } \\
\text { e ansiedade materna. }\end{array}$ \\
\hline Robinson et al. ${ }^{9}$ & $\begin{array}{l}\text { Mães de crianças com fracasso no desenvolvimento produzem menos } \\
\text { estratégias de resolução de problemas. }\end{array}$ \\
\hline Weiss $^{10}$ & $\begin{array}{l}\text { Mães de crianças com autismo mostraram níveis mais altos de ansiedade e } \\
\text { depressão. }\end{array}$ \\
\hline Olsson et al. ${ }^{11}$ & $\begin{array}{l}\text { Mães de crianças com autismo experienciavam mais estresse frente aos } \\
\text { sintomas do autismo e maior risco para desenvolver depressão. }\end{array}$ \\
\hline Solomon et al. ${ }^{12}$ & $\begin{array}{l}\text { Mães de crianças com autismo que receberam intervenção apresentaram } \\
\text { diminuição nos escores de depressão. }\end{array}$ \\
\hline Carte & Mães de crianças com autismo apresentaram elevados sintomas depressivos. \\
\hline Roncol & $\begin{array}{l}\text { Uma mãe de uma criança com autismo com depressão pós-parto recebeu suporte } \\
\text { psico-social. Tanto a mãe quanto a criança apresentaram melhoras. }\end{array}$ \\
\hline Wachtel e Carter ${ }^{15}$ & $\begin{array}{l}\text { Mães que receberam intervenção apresentaram maior aceitação do diagnóstico } \\
\text { de seus filhos e estilo de parentalidade mais positivo. }\end{array}$ \\
\hline Kersh et al. ${ }^{16}$ & $\begin{array}{l}\text { Os pais com maior qualidade marital apresentavam menos estresse e menos } \\
\text { sintomas depressivos. }\end{array}$ \\
\hline Mickelson et al. ${ }^{17}$ & $\begin{array}{l}\text { Os pais que atribuíram a condição da criança à própria culpa e ao ambiente } \\
\text { apresentaram um pior ajustamento (autismo). Já os pais que atribuíram ao } \\
\text { acaso genético e ao destino apresentaram um melhor ajustamento parental } \\
\text { (SD). }\end{array}$ \\
\hline
\end{tabular}

ginam diferentes expectativas e necessidades ou formas de superação para as famílias. ${ }^{7,8} \mathrm{~A}$ sobrecarga está relacionada ao excesso de exigências, de cuidados e de tarefas peculiares e pode resultar em estresse e tensão emocional, colocando os pais e, em especial, as mães, em risco de desenvolver depressão ${ }^{4}$. Isto porque ao se comparar o estado emocional dos pais e das mães de crianças com autismo encontrou-se que as mães apresentam maior incidência de estresse $^{5}$ e depressão, possivelmente porque a mãe, em geral, é a responsável pela maior parte dos cuidados diretos da criança. ${ }^{4}$

Dos estudos revisados alguns investigaram o impacto de ter um filho com autismo na saúde mental materna. Para esta investigação os autores compararam mães de crianças com autismo com mães de crianças portadoras de outras patologias ou ainda com mães de crianças com Desenvolvimento Típico (DT). ${ }^{9,10,11,12,13} \mathrm{O}$ estudo de Robinson et al. ${ }^{9}$ encontrou que mães de crianças com atrasos no desenvolvimento e com autismo apresentaram risco mais elevado para desenvolver depressão e foram mais severamente afetadas por este quadro clínico. Além disso, essas mães desenvolveram menos estratégias de enfrentamento de problemas do que as mães de crianças com DT, possivelmente porque apresentavam um repertório insuficiente e pobre na qualidade de suas respostas, levando, assim, a uma maior frustração no seu papel materno, aumentando 
o conflito na interação com a criança e diminuindo o seu senso de competência.

Neste sentido, o estudo de Weiss ${ }^{10} \mathrm{com}$ parou mães de crianças autistas com mães de crianças com deficiência mental e mães de crianças com DT. Já Olsson et al. ${ }^{11}$ compararam mães de crianças com autismo ou com DM, com mães de crianças com DT. Os resultados destes estudos revelaram uma prevalência significativamente maior de depressão e ansiedade nas mães de crianças com autismo, quando comparadas às mães de crianças com DT ou com outras patologias. Isto ocorre, principalmente, devido aos problemas desenvolvimentais típicos do autismo. ${ }^{10,11}$ Dentre eles, destacam-se: atraso no funcionamento cognitivo, na maioria dos casos, dificuldades na comunicação verbal e não-verbal e nas relações interpessoais. As interações sociais da criança tendem a ser pouco recíprocas e espontâneas; podem responder às solicitações dos outros, mas raramente iniciam e mantêm a interação, de forma frequente e variada. A frustração materna decorrente desta característica, somada à dificuldade de comunicar desejos e intenções, é bem documentada na literatura ${ }^{10,11}$.

Esses fatores podem estar implicados na origem da depressão materna, embora não a explique completamente. Por exemplo, Olsson et al. ${ }^{11}$ constataram que mães de crianças com deficiência (com ou sem autismo), que viviam sozinhas, eram mais vulneráveis a apresentar depressão do que as mães que viviam com um companheiro. Portanto, ainda que o autismo apresente um grande desafio à maternagem, há outros fatores que podem intensificar o estresse. De fato, os autores verificaram que as mães de crianças com autismo com maior nível de escolaridade utilizaram melhor os recursos para procurar ajuda. Neste caso, o suporte social e a escolaridade foram fatores mediadores do estresse materno e favoreceram o ajustamento familiar como um todo ${ }^{10,11}$. Os estudos apontam que baixos níveis de suporte social são os preditores mais fortes para depressão e ansie- dade, ${ }^{8,13,16}$ que a diminuição dos escores de depressão estão relacionados a intervenções que oferecem apoio social ${ }^{12,14} \mathrm{e}$, por fim, que a intervenção mais adequada no contexto do autismo depende da fase de desenvolvimento em que a criança está, bem como do contexto familiar. $^{7}$

Por outro lado, estudos revelam que mães que vivem situações semelhantes, como o fato de ter um filho com desenvolvimento atípico, nem sempre são afetadas da mesma maneira, ainda que estejam diante desta sobrecarga $^{5}$. Apesar da prevalência de depressão materna variar entre os estudos, ${ }^{12,13}$ é evidente que muitas mães de crianças com deficiência não sofrem de depressão. Uma das explicações encontradas para esta questão baseia-se nas teorias cognitivas da depressão, ${ }^{17}$ que reconhecem a importância dos eventos estressores para o início e o curso de um quadro depressivo. $\mathrm{O}$ que não quer dizer que a presença de um evento estressor, por si só, desencadeie um quadro depressivo, já que a maioria dos indivíduos não desenvolve depressão quando se depara com experiências de vida difíceis e estressantes. Isso porque o que determinará o impacto dessas experiências sobre o indivíduo será o significado atribuído pela pessoa à experiência. Se um evento de vida ou estressor relaciona-se a questões pessoais ou preocupações que são centrais para a auto-percepção do indivíduo, então uma resposta depressiva poderá ser eliciada.

Desta forma, esquemas disfuncionais pré-existentes podem fazer alguns pais particularmente sensíveis a desenvolver sentimentos de perda, desesperança e de falha, especialmente se eles não têm uma ativação adequada de esquemas compensatórios positivos ${ }^{17}$. Pais de crianças com deficiência provavelmente não apresentam mais esquemas disfuncionais do que pais de crianças com DT, antes do nascimento da criança. Porém, os esquemas disfuncionais que permanecem inativos nos pais de crianças com DT têm maior possibili- 
dade de serem ativados nos pais de crianças com deficiência. ${ }^{17}$

A relação entre um evento estressante $\mathrm{e}$ um esquema disfuncional pode não ser a única razão para que as mães de crianças com deficiência desenvolvam depressão. Parece razoável supor que algumas dessas mães teriam sido deprimidas, mesmo na ausência da deficiência de seu filho, por causa de suas próprias vulnerabilidades biológicas ou emocionais. Para outras mães, existe uma vulnerabilidade cognitiva que é desencadeada diante dos múltiplos estressores envolvidos na experiência de ter um filho com desenvolvimento atípico. $\mathrm{Na}$ ausência de uma criança com deficiência, esses esquemas disfuncionais poderiam ter sido ativados por algum outro estressor, ou poderiam ter permanecido desativados ${ }^{17}$.

De acordo com Olsson et $\mathrm{al}^{11}$. mais estudos seriam necessários a fim de verificar as possíveis diferenças psicológicas entre mães deprimidas de crianças com desenvolvimento atípico. Tais estudos precisariam investigar a saúde mental dessas mães antes do nascimento da criança, a história de depressão na família, a gravidade e a duração da depressão e as circunstâncias que rodearam o aparecimento da depressão, assim como descrições qualitativas e análise dos pensamentos e sentimentos suscitados nas mães em diferentes situações.

\section{CONSIDERAÇÕES FINAIS}

Com base na revisão realizada foi possível verificar que os níveis de depressão são elevados entre mães de crianças com transtornos do desenvolvimento, como no caso do autismo. Um fator que pode influenciar os quadros depressivos relaciona-se às próprias características do autismo, como a dificuldade na interação social, que exige das mães um manejo diferenciado para lidar com a situação, para a qual geralmente elas não estão preparadas. Além disso, a saúde mental das mães, anterior ao nascimento de uma criança com autismo, deve ser considerada, uma vez que mães predispostas a desenvolverem depressão poderiam ter esses sintomas desencadeados por outro fator. Porém, os processos depressivos de mães de crianças com autismo ainda são pobremente compreendidos, devido à escassez de estudos que envolvem essa temática.

No entanto, mesmo havendo poucos estudos - o que dificulta avanços na criação de programas que auxiliem mais eficazmente mães nessas condições - medidas devem ser tomadas para auxiliar essas mães em uma situação que coloca em risco sua saúde mental e, assim, minimizar o impacto da depressão materna no desenvolvimento infantil. Dentre essas medidas destaca-se a importância da presença do pai ou de outro adulto, não deprimido, bem como o uso de intervenções de suporte social. Este último aspecto tem sido considerado um dos fatores-chave para o abrandamento do estresse - e, consequentemente, do risco para desenvolver depressão, uma vez que a satisfação das mães com o apoio percebido e recebido tem resultado em um melhor bem-estar pessoal e em uma relação mais positiva com o filho, minimizando, assim os efeitos negativos dos sintomas depressivos, tanto para a saúde mental materna como para o desenvolvimento infantil.

Pesquisas futuras devem verificar como o uso de uma rede de apoio social pelas mães resulta em comportamentos mais favorávies tanto para ela quanto para o filho, assim como que tipo de apoio é mais eficaz no alívio dos sintomas depressivos. A partir destes resultados, os profissionais desta área poderão contar com mais recursos para entender e atender as necessidades maternas e ajudá-las a adaptarem-se às situações desafiadoras que envolvem a criação e educação de uma criança com autismo. 


\section{REFERÊNCIAS}

1. Brum E, Schermann L. O impacto da depressão materna nas interações iniciais. Psico. 2006; 37(2): 151-158.

2. Moraes I, Pinheiro R., Silva R., Horta B, Souza P, Faria A. Prevalência da depressão pós-parto e fatores associados. Rev Saúde Públ. 2006; 40(1): 65-70.

3. Sanini C, Ferreira G, Souza T, Bosa C. Comportamentos indicativos de apego em crianças com autismo. Psicol. Reflex. Crit. 2008; 21(1): 60-65.

4. Hastings R, Kovshoff H, Ward N, DegliEspinosa F, Brown T, Remington B. Systems Analysis of Stress and Positive Perceptions in Mothers and Fathers of PreSchool Children with Autism. J Autism Dev Disord. 2005; 35(5): 635-644.

5. Favero M, Santos M. Autismo infantil e estresse familiar: uma revisão sistemática da literatura. Psicol. Reflex. Crit. 2005; 18(3): 358-369.

6. Fernandes F. Famílias com crianças autistas na literatura internacional. 2009; Rev Soc Bras Fonoaudiol. 2009; 14(3): 427-32.

7. Bosa C. Autismo: intervenções psicoeducacionais. Rev Bras Psiquiatr. 2006; (28): 47-53.

8. Boyd B. Examining the relationship between stress and lack of social support in mothers of children with autism. Focus on Autism and Other Develop Disab. 2002; 17(4): 208-215.

9. Robinson J, Drotar D, Boutry M. Problemsolving abilities among mothers of infants with failure to thrive. J Pediatr Psychol. 2001; (1): 21-32.
10. Weiss MJ. Hardiness and social support as predictors of stress in mothers of typical children, children with autism and children with mental retardation. Autism. 2002; 6(1): 115-130.

11. Olsson MB, Hwang CP. Depression in mothers and fathers of children with intellectual disability. J Intellect Disabil Res. 2001; 45(6): 535-543.

12. Solomon M, Goodlin-Jones B, Anders T. A Social Adjustment Enhancement Intervention for High Functioning Autism, Asperger's Syndrome, and Pervasive Developmental Disorder NOS. J Autism Dev Disord. 2004; 34(6): 649-668.

13. Carter A, Martínez-Pedraza F, Gray S. Stability and individual change in depressive symptoms among mothers raising young children with ASD: Maternal and child correlates. J Clin Psychol. 2009; 65(12): 1270-1280.

14. Roncon P. Abordagens familiares face ao autismo. Análise Psicológica. 2003; 21(1): 53-57.

15. Wachtel K, Carter A. Reaction to diagnosis and parenting styles among mothers of young children with ASDs. Autism. 2008; 12(5): 575-594.

16. Kersh J, Hedvat T, Hauser-Cram P, Warfield M. The contribution of marital quality to the well-being of parents of children with developmental disabilities. J Intellect Disabil Res. 2006; 50(12): 883893.

17. Mickelson KD, Wroble M, Helgeson VS. 'Why my child?': Parental attributions for children's special needs. J Appl Soc Psychol. 1999; 29(6): 1263-1291.

Recebido em 02/02/10 Modificado em 06/07/10 Aceito em 12/08/10 